\title{
The relationship between corticospinal excitability during motor imagery and motor imagery ability
}

\author{
${ }_{4}$ Q1 Jacqueline Williams ${ }^{\mathrm{a}, \mathrm{b}, \mathrm{c}, *}$, Alan J. Pearce ${ }^{\mathrm{b}}$, Michela Loporto $^{\mathrm{c}}$, Tony Morris ${ }^{\mathrm{a}, \mathrm{b}}$, Paul S. Holmes $^{\mathrm{c}}$ \\ a Institute for Sport, Exercise and Active Living, Victoria University, PO Box 14428, Melbourne, VIC, 8001, Australia \\ ${ }^{\mathrm{b}}$ School of Sport and Exercise Science, Victoria University, PO Box 14428, Melbourne, VIC, 8001, Australia \\ ' Institute for Performance Research, Manchester Metropolitan University, Cheshire Campus, Crewe Green Road, Crewe, Cheshire, CW1 5DU, United Kingdom
}

\section{A R T I C L E I N F O}

\section{Article history:}

Received 29 July 2011

Received in revised form 6 September 2011 Accepted 7 September 2011

Available online $\mathrm{xxx}$

\section{Keywords:}

Motor imagery

Action observation

Transcranial magnetic stimulation

Imagery ability

\begin{abstract}
A B S T R A C T
It is commonly reported that transcranial magnetic stimulation (TMS) of the motor cortex during action observation and motor imagery results in increases in the amplitude of motor evoked potentials (MEPs) in muscles specific to the observed or imagined action. This study aimed to determine whether MEP amplitude was related to the motor imagery ability of participants. Participants were 15 healthy, righthanded adults (five male), with a mean age of 29.7 years. Motor imagery ability was measured using the Vividness of Movement Imagery Questionnaire-2 (VMIQ-2) and a hand rotation task. TMS was delivered during observation and imagery of a finger-thumb opposition sequence and MEPs were measured in the abductor pollicis brevis. Significant increases in MEP amplitude, from baseline, were recorded during observation and imagery conditions. The change in amplitude to both observation and imagery was expressed as a percentage of baseline amplitude. There was a significant correlation between MEP change for the imagery condition and imagery ability, with greater change linked to more vivid images and faster response times. The relationship between MEP change for the observation condition and imagery ability was less salient. This is the first study to show that the strength of corticospinal activation during imagery, which may be a determinant of the effectiveness of imagery training, is related to imagery ability in the general population, and has implications for clinical programs.
\end{abstract}

(C) 2011 Published by Elsevier B.V.

\section{Introduction}

Action observation and motor imagery have been flagged as potentially useful tools in rehabilitation from stroke and other brain injuries [1,2]. Improved methods to measure brain activity, including functional magnetic resonance imagining (fMRI) and transcranial magnetic stimulation (TMS), have shown that motor imagery and observation of actions, can, when used effectively, activate the neural motor system in a way that overlaps significantly with that activated during actual movement [3-7]. When physical movement is impossible, or perhaps very difficult, in the early stages following a brain injury, observation of relevant movements and task-specific motor imagery offer potential avenues to activate the neural motor system without physical movement needing to occur and is, therefore, not reliant on there being some level of residual function [1]. This early activation, specifically in areas of localised damage, may ensure that neuronal function is

\footnotetext{
* Corresponding author at: Institute for Sport, Exercise and Active Living, Victoria University, PO Box 14428, Melbourne, VIC, 8001, Australia. Tel.: +61 39919 4025; fax: +61 399194891.

E-mail address: jacqueline.williams@vu.edu.au (J. Williams).
}

not lost as a result of inactivity that may otherwise result from the lack of proprioception from significantly reduced physical movement. Although theoretically exciting, the imagery interventions are not always successful [e.g. 8], and at this stage, the factors that may affect the success of such interventions have not been clearly defined.

One potentially influential factor may be the ability of the affected individual to form vivid motor images. This ability varies as a result of a number of factors, including motor experience, age and gender [9]. It seems intuitive that the extent of the vividness of a motor image would be associated with the pattern and/or level of neural activation in motor and related areas but, somewhat surprisingly, this relationship has received little attention from researchers. Studies in sport psychology suggest that athletes who utilise motor imagery regularly and report higher levels of imagery vividness for their sport specific tasks demonstrate different patterns of neural activation to novice or non-athlete groups who use motor imagery less $[10,11]$. Skilled performers also demonstrate significantly higher levels of corticospinal activation following TMS during imagery of movements related to their particular sport, when compared to novice performers [12]. However, these differences dissipate when assessing imagery of more general motor skills, unrelated to their sport $[10,12]$. It has been demonstrated

0166-4328/\$ - see front matter (c) 2011 Published by Elsevier B.V. doi:10.1016/j.bbr.2011.09.014 
that intensive physical practice and subsequent improvement in the performance of a motor skill also alters the pattern of neural activation during motor imagery [13], and taken with our knowledge of the imagery ability of elite athletes, this indicates that task experience can influence imagery ability and accompanying neural activation. However, it is unclear whether the differences in activation in athletes compared to novice performers are related to the vividness of the motor images per se or that the changes in neural activation have occurred as a result of motor learning.

In their study, Fourkas et al. [12] required both expert and novice tennis players to complete the Movement Imagery Questionnaire - revised [MIQ-R; 14]. The amplitude of the motor evoked potential (MEP) resulting from TMS during imagined tennis movements was significantly correlated with the visual and kinaesthetic imagery scores from the MIQ-R in the sample as a whole $(r=.52$ and .68 respectively), with more vivid imagery scores resulting in greater MEP amplitude. The observed relationship was stronger in the expert group than the novice group, highlighting the need for studies using observation and imagery of less specialised skills.

To the best of our knowledge, only one study has been conducted that specifically addresses the issue of neural activation and motor imagery ability in the general population. Guillot et al. [15] used a comprehensive battery of motor imagery tasks to classify typical adults into two groups - skilled and unskilled imagers. Participants were then required to undergo an fMRI scan, during which they had to imagine executing a pre-learned finger movement sequence. Both groups showed activation in motor related regions and the inferior and superior parietal lobes. However, when the activation patterns of the groups were contrasted, the skilled imagers recorded greater activation in parietal and premotor areas, both of which play crucial roles in motor imagery [16,17]. In comparison, the poor imagers recorded greater activation in posterior cingulate and orbito-frontal cortices (memory-related rather than imagery-related areas) and also in areas of the cerebellum. This is the first paper to demonstrate that, in the general population, motor imagery ability impacts upon neural activation during motor imagery.

The current study aimed to determine whether there was a significant relationship between motor imagery ability and MEP amplitude following TMS during motor imagery. It has commonly been demonstrated that MEP amplitude in muscles specific to the imagined movement increases during motor imagery (and movement observation) when compared to rest conditions [e.g. $6,18,19]$. Though lower in amplitude than during actual movement, the increased MEPs during imagery and observation, relative to baseline, provide evidence that both techniques activate the corticospinal pathways in a similar way to actual movement. Recent studies have begun to explore factors that might affect the amplitude of MEPs observed, including task complexity and mode of imagery $[20,21]$, but none have yet considered motor imagery ability. Therefore, in this study, we first measured motor imagery ability using two commonly used techniques - a self-report questionnaire; the Vividness of Movement Imagery Questionnaire-2 (VMIQ-2) [22] and the hand rotation task [23]. We confirmed that MEP amplitude recorded from the abductor pollicis brevis (APB) muscle was greater during action observation and motor imagery than the baseline condition, and correlated change in MEP amplitude from baseline with measures of imagery ability.

In line with Clark et al. [6], we hypothesized that the MEP amplitude during action observation and motor imagery would not differ, but that MEP amplitude during both conditions would be significantly greater than that obtained during baseline. Physical movement was hypothesized to produce the greatest MEP amplitude and result in significantly reduced MEP latency periods than all other conditions. Recent findings have revealed greater MEP amplitude during kinaesthetic motor imagery compared to visual motor imagery $[7,20]$. Therefore we expected to find a significant inverse correlation between change in MEP amplitude and kinaesthetic imagery scores from the VMIQ-2, but not for the visual imagery scores. We also expected to find a significant inverse correlation between the change in MEP amplitude and hand rotation performance.

\section{Method}

\subsection{Participants}

Fifteen healthy young adults, with a mean age of 29.67 years $(S D=5.29$; five males) were recruited through the University setting to participate in this study. All participants were right-handed, as determined using the Edinburgh Inventory [24] and were screened to ensure that they did not have any current or past neurological conditions, were not pregnant, and did not have any implants (e.g., pacemakers, cochlear implants) that may be affected by TMS. No participants were excluded on this basis. No participants were competing in elite level sports, and their motor abilities are described briefly in Section 3.

\subsection{Measures}

\subsubsection{McCarron assessment of neuromuscular development [MAND; 25]}

The MAND is a standardised motor assessment battery used here to describe the motor skill level of participants. With normative data for both children and adults, the MAND consists of 10 motor tasks (five fine motor, five gross motor), scores for which are standardised and summed to determine the overall Neuromuscular Development Index $(M=100 ; \mathrm{SD}=15)$.

\subsubsection{Vividness of Movement Imagery Questionnaire-2 [VMIO-2; 22]}

The VMIQ-2 requires participants to imagine performing 12 movements using (1) external visual imagery (watching yourself perform from an external viewpoint) (2) internal visual imagery (imaging as if you were looking out through your own eyes as you move) and (3) kinaesthetic imagery (where the focus is on feeling yourself perform the movement). Participants rated the vividness of each image using a 5-point Likert scale, with scores from each item summed to provide a vividness score for each component of between 12 and 60 (with lower scores indicating more vivid images). The factorial, concurrent and construct validity of the VMIQ-2 has previously been demonstrated [22].

\subsubsection{Hand rotation task}

Single hand stimuli $(9 \mathrm{~cm} \times 8 \mathrm{~cm})$ were presented on a laptop computer using EPrime software (Psychology Software Tools, Pittsburgh, PA, USA). Participants were required to decide whether each presented stimulus was a left or a right hand as quickly and accurately as possible. The hands were high-resolution images presented in both the palm and back view (see Fig. 1), centred in the middle of the screen. Hands were presented randomly in $45^{\circ}$ increments between 0 and $360^{\circ}$ and remained on screen until a response was recorded by pressing a designated key on the computer keyboard or $10 \mathrm{~s}$ had passed. Responses were recorded to the nearest $1 \mathrm{~ms}$. Participants completed five practice trials followed by 80 test trials, providing five trials in each view at each angle.

\subsection{Procedure}

The project protocol was approved by the Human Research Ethics Committee at Victoria University, Melbourne. After providing informed consent, participants completed two assessment sessions on different days. The first session involved a one-on-one movement imagery assessment, where the participants completed the hand rotation task and the VMIQ-2. In the second session, participants completed the TMS protocol. This protocol involved the identification of each participant's optima scalp position (OSP) and resting motor threshold, followed by 10 randomly timed single TMS pulses during each of the following conditions: (1) baseline (static hand) (2) movement observation; (3) movement imagery; and (4) actual movement.

Participants were required to sit in a relaxed position in front of a $15.4 \mathrm{in}$ computer screen, positioned $.5 \mathrm{~m}$ away. During the baseline condition, participants watched video footage of a static hand resting on a table-top. During movement observation, participants watched video footage of the same hand performing a continuous finger-thumb opposition task. Movements were paced so that one cycle (from index to little finger) took $4 \mathrm{~s}$. Participants were then asked to keep their eyes open and imagine themselves performing the same task that they had just observed, with an emphasis on feeling the movement (movement imagery condition). In the final condition, participants actually performed the task (actual movement condition). Each condition lasted approximately $1 \mathrm{~min}$.

Electromyographic (EMG) readings were collected from the APB muscle of the right hand using surface electrodes. TMS was performed using a MagStim $200^{2}$ magnetic stimulator (Magstim Co., Whitland, Dyfed, UK) and a hand-held $70 \mathrm{~mm}$ figure of eight coil. Participants wore a cap that was pre-marked in $1 \mathrm{~cm}$ increments in both lateral and anterior-posterior directions, positioned in reference to the nasion-inion and interaurel lines. The coil was held over the left motor cortex, 
in an anterior-posterior orientation tangential to the skull, over the OSP for stimulating the APB muscle. The OSP was identified for each participant by exploring sites near the estimated centre of the hand area (beginning $6 \mathrm{~cm}$ lateral to the vertex) using a stimulus intensity of $60 \%$. OSP was considered to be the site at which the largest MEP could be obtained consistently. Resting motor threshold (RMT) was determined by stimulating the OSP while the participant rested their hand on their lap. Stimulations began at 30\% and were increased in 5\% increments until a subject recorded at least 5 of $10 \mathrm{MEP}$ peak-to-peak amplitudes greater than $50 \mu \mathrm{V}$. For test conditions, intensity was set at $10 \%$ of stimulator output above the RMT for each subject. EMG activity was amplified $(\times 1000)$ and digitised for $500 \mathrm{~ms}$ following each stimulus. Stimulations were delivered manually at $6 \mathrm{~s}$ intervals. Waveforms were stored offline for further analysis.

\subsection{Data analysis}

\subsubsection{Motor imagery data}

For each participant, item scores within each component of the VMIQ-2 were summed to provide vividness ratings for 'internal' and 'external' visual and kinaesthetic imagery. A repeated measures ANOVA was conducted to determine whether participant's ability to produce vivid images depended upon the mode of imagery used.

Mean RT and accuracy for each hand at each angle of rotation was calculated for each participant. No anticipatory (less than $250 \mathrm{~ms}$ ) or significantly delayed (2.5 times greater than the mean for that angle) responses were identified. Initially, mean RTs to hand stimuli rotated medially (for left hands, this included hands at angular rotations of $45^{\circ}, 90^{\circ}$ and $135^{\circ}$, and for right hands, $315^{\circ}, 270^{\circ}$ and $225^{\circ}$ ) were compared to hand stimuli rotated laterally (left hands at $315^{\circ}, 270^{\circ}$ and $225^{\circ}$ and right hands at $45^{\circ}, 90^{\circ}$ and $135^{\circ}$ ) to determine whether responses of the sample conformed to the biomechanical constraints of real movements, thereby supporting the use of movement imagery. A paired-sample $t$-test was conducted to determine if RT to medial and laterally rotated hands differed significantly. Secondly, to allow
Table 1

Motor imagery performance measures.

\begin{tabular}{llc}
\hline Measure & Component & Mean (SD) \\
\hline VMIQ-2 & External visual imagery & $27.47(12.95)$ \\
& Internal visual imagery & $24.93(10.66)$ \\
& Kinaesthetic imagery & $23.00(10.10)$ \\
Hand rotation & Response time (all angles) & $1581.96(466.28)$ \\
& Response time (medial rotations) & $1370.39(446.15)$ \\
& Response time (lateral rotations) & $1629.77(560.79)$ \\
& Response accuracy (all angles) & $90.50 \%(5.80)$ \\
\hline
\end{tabular}

Note: Response time is in ms; accuracy is percentage of correct responses.

correlations with the TMS data, each individual's mean RT and accuracy across all angles was calculated.

\subsubsection{TMS/EMG data}

The MEP peak-to-peak amplitude ( $\mu \mathrm{V})$ and TMS latency (ms) was extracted manually for each of the 10 trials in each condition for all participants. Mean amplitude and latency for each of the four conditions was then calculated. In line with Clark et al. [6], amplitude data was transformed using a natural logarithm to normalise data. Repeated measures ANOVAs were then conducted on log-amplitude and latency data, with four within-subject variables: (1) baseline (static hand); (2) movement observation; (3) movement imagery; and (4) actual movement. A significant effect for condition was followed by multiple pairwise comparisons of estimated marginal means with Bonferroni adjustments.

To determine the change in amplitude during the observation and imagery conditions from baseline, we calculated the difference in amplitude between the two conditions and expressed this as a percentage of the baseline amplitude [see also 26]. These values were then submitted to a bivariate correlation analysis with each
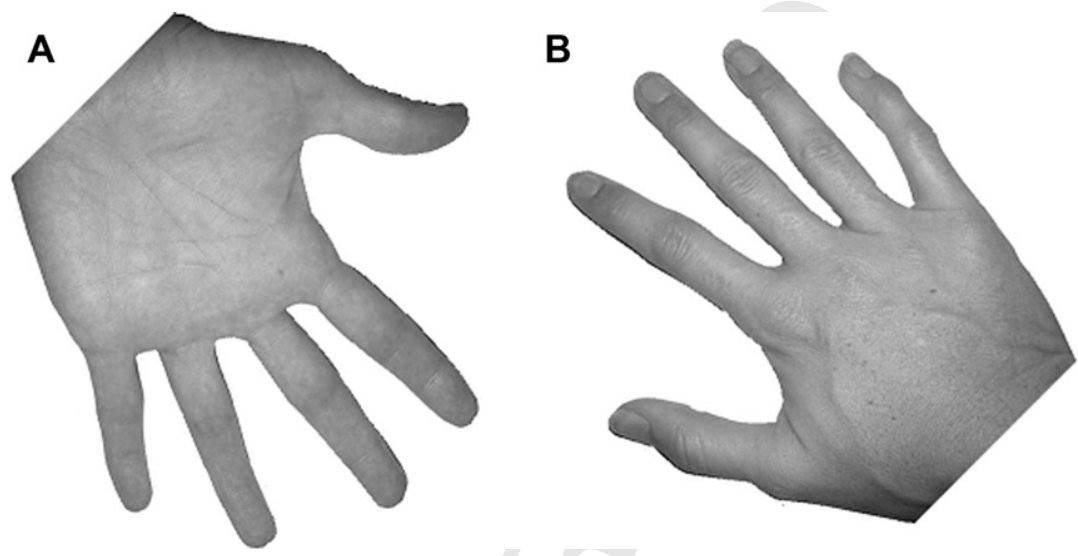

Fig. 1. Hand rotation task stimuli. Left hand in palm view at $135^{\circ}$ and right hand in back view at $315^{\circ}$.

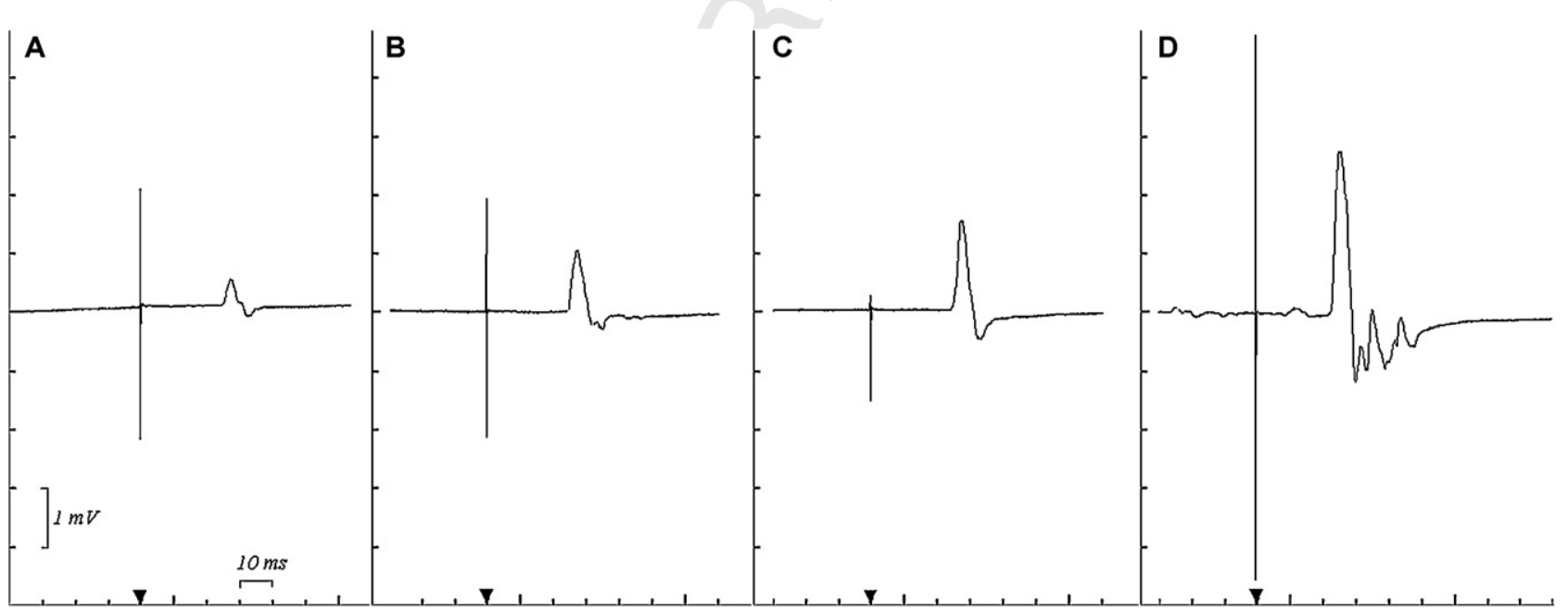

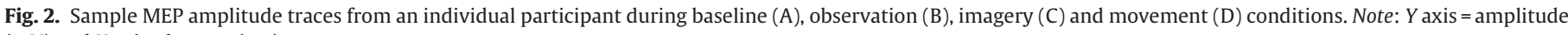
$(\mathrm{mV})$ and $\mathrm{X}$ axis $=$ latency $(\mathrm{ms})$. 

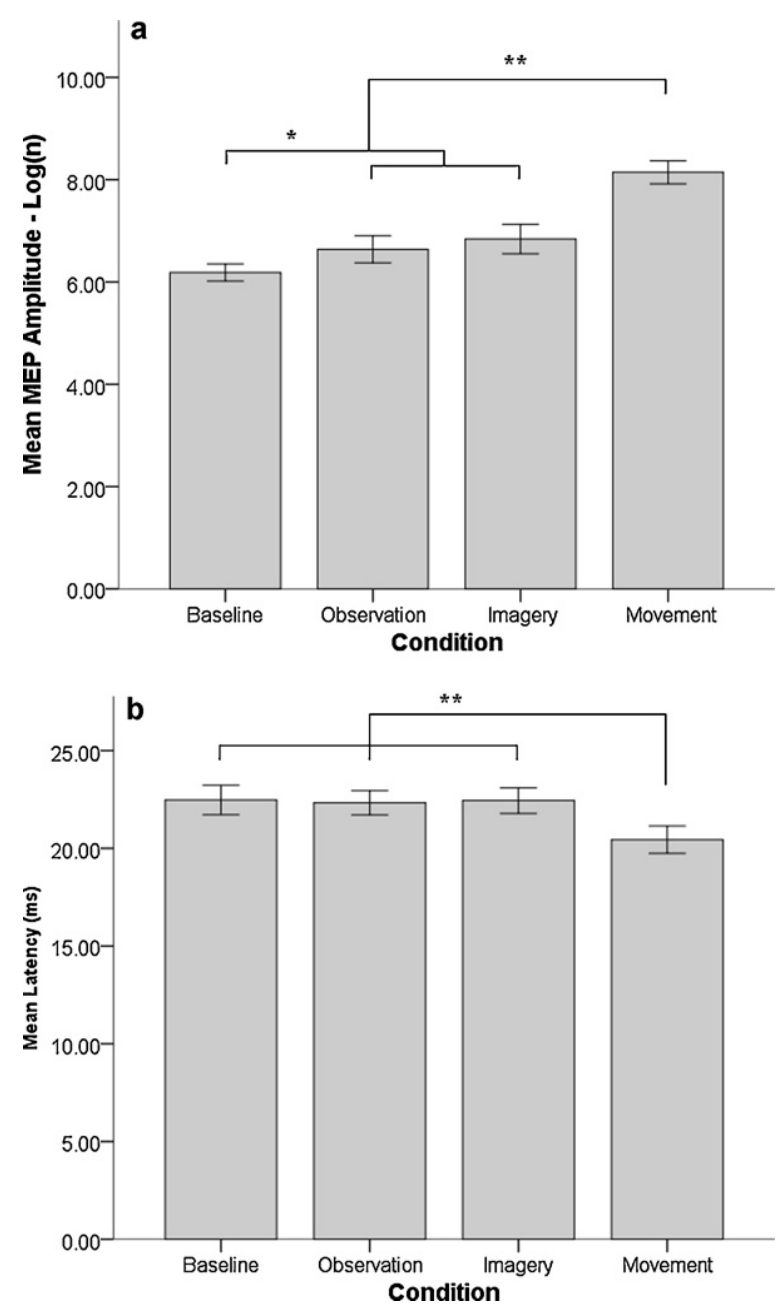

Fig. 3. MEP amplitude (a) and latency (b) during the four conditions. Note: ${ }^{*} p<.05$; ${ }^{* *} p<.001$.

component score from the VMIQ-2 and mean RT and accuracy from the hand rotation task. Correlations were interpreted using Cohen's [27] guidelines, where $r$ of $<.3$ is small, $.3-.5$ is medium and $>.5$ is large. All statistical analyses were conducted using SPSS PASW Statistics 18.

\section{Results}

The results of the MAND indicated that all participants had motor skills that were within age expected levels. The mean score for the Neuromuscular Development Index was 96.90 ( $S D=10.56$ ), with scores ranging from 74 to 116 . Descriptive results for the motor imagery tasks are presented in Table 1 . There was no significant difference in vividness scores among the three modalities of imagery, $F(2,26)=.77, p=.48, \eta_{\mathrm{p}}^{2}=.06$. Responses to hands rotated medially were significantly faster than responses to hands rotated laterally, $t(14)=3.88, p=.002$, indicating responses were constrained by the biomechanical limitations of real movements.

An example of a participant's MEP amplitude trace for each of the conditions is shown in Fig. 2, and sample mean amplitude and latency data for each condition can be seen in Fig. 3. There was a significant effect of condition on movement amplitude, $F(3,42)=65.38, p<.001, \eta_{\mathrm{P}}^{2}=.82$. Post-hoc tests indicated that MEP amplitude during the baseline condition was significantly lower than during the observation, imagery and actual movement conditions ( $p=.012, .005$ and $<.001$ respectively). MEP amplitude did not differ between the observation and imagery conditions $(p=1.00)$, but was significantly greater during actual movement $(p<.001)$. There was a significant effect of condition on MEP latency, $F(3,42)=35.58, p<.001, \eta_{\mathrm{p}}^{2}=.72$. MEP latency was significantly reduced in the movement condition compared to the other three conditions (all $p<.001$ ). There were no other significant differences.

For the observation condition, MEP amplitude increased, on average, by $73.72 \%(S D=82.88)$ from the baseline condition; for the imagery condition, this increase was $127.03 \%(S D=135.21)$ of the baseline condition. One participant was a substantial outlier, with an increase from baseline to imagery of 630\%. This participant was excluded from the correlation analysis. The correlations between the motor imagery measures and the change in MEP amplitude during observation and imagery are shown in Table 2 and scatterplots for correlations between kinaesthetic imagery scores/mean hand task RT and \% change in MEP amplitude during imagery/observation can be seen in Fig. 4. Change in MEP amplitude during the imagery condition demonstrated a large and significant inverse correlation with the kinaesthetic component of the VMIQ-2 and average RT for the hand task. Change in MEP amplitude during the observation condition was not as strongly correlated with the motor imagery measures, demonstrating only medium, non-significant correlations.

\section{Discussion}

The major aim of this study was to determine whether MEP amplitude following TMS during motor imagery was correlated with motor imagery ability. The results showed a strong and significant correlation between selected measures of motor imagery and the change in MEP amplitude from baseline to imagery. The relationship between motor imagery measures and the change in MEP amplitude from baseline to observation was less salient. These findings have implications for clinical interventions, which will be discussed later. Next, we review the behavioural and MEP findings.

\subsection{Behavioural findings}

The VMIQ-2, a popular measure in sport psychology, provides a rating of imagery vividness from external and internal perspectives for visual and kinaesthetic imagery. Vividness ratings across the three components of the VMIQ-2 did not differ significantly, though it is interesting to note that kinaesthetic imagery was rated as the most vivid.

Although the hand rotation task does not provide explicit imagery instructions, participants usually report imagining their own hands in the position of the hand on the screen to make the required laterality decision [28]. Supporting the use of motor imagery to perform this task, neuroimaging studies confirm that this task activates the motor areas of the brain [e.g. 29,30], while others have shown that responses to medially rotated stimuli are faster than those to laterally rotated stimuli, reflecting the biomechanical constraints of real movement [31,32]. In the current sample, response times to medially rotated stimuli were significantly faster than responses to laterally rotated stimuli and as such, we are confident that motor imagery was being performed.

\subsection{MEP findings}

As predicted, and in line with Clark et al. [6] who used a similar study design, we found a significant increase in the amplitude of MEPs during the motor imagery and observation conditions compared with baseline, with a further significant increase during actual movement. In regard to MEP latency, only actual movement produced a significant reduction, which also follows the findings of Clark et al. [6]. These results add to the growing body of evidence demonstrating increases in corticospinal excitability during 
Table 2

Correlations among imagery measures and the change in MEP amplitude.

\begin{tabular}{|c|c|c|c|c|c|}
\hline \multirow[t]{2}{*}{ MEP change } & \multicolumn{5}{|l|}{ Imagery variable } \\
\hline & External visual imagery & Internal visual imagery & Kinaesthetic imagery & Hand task RT (total) & Hand task accuracy \\
\hline Baseline to imagery & .04 & -.44 & $-.65^{*}$ & $-.56^{*}$ & .31 \\
\hline Baseline to observation & -.11 & -.16 & -.35 & -.46 & .33 \\
\hline
\end{tabular}

* $p<.05$.
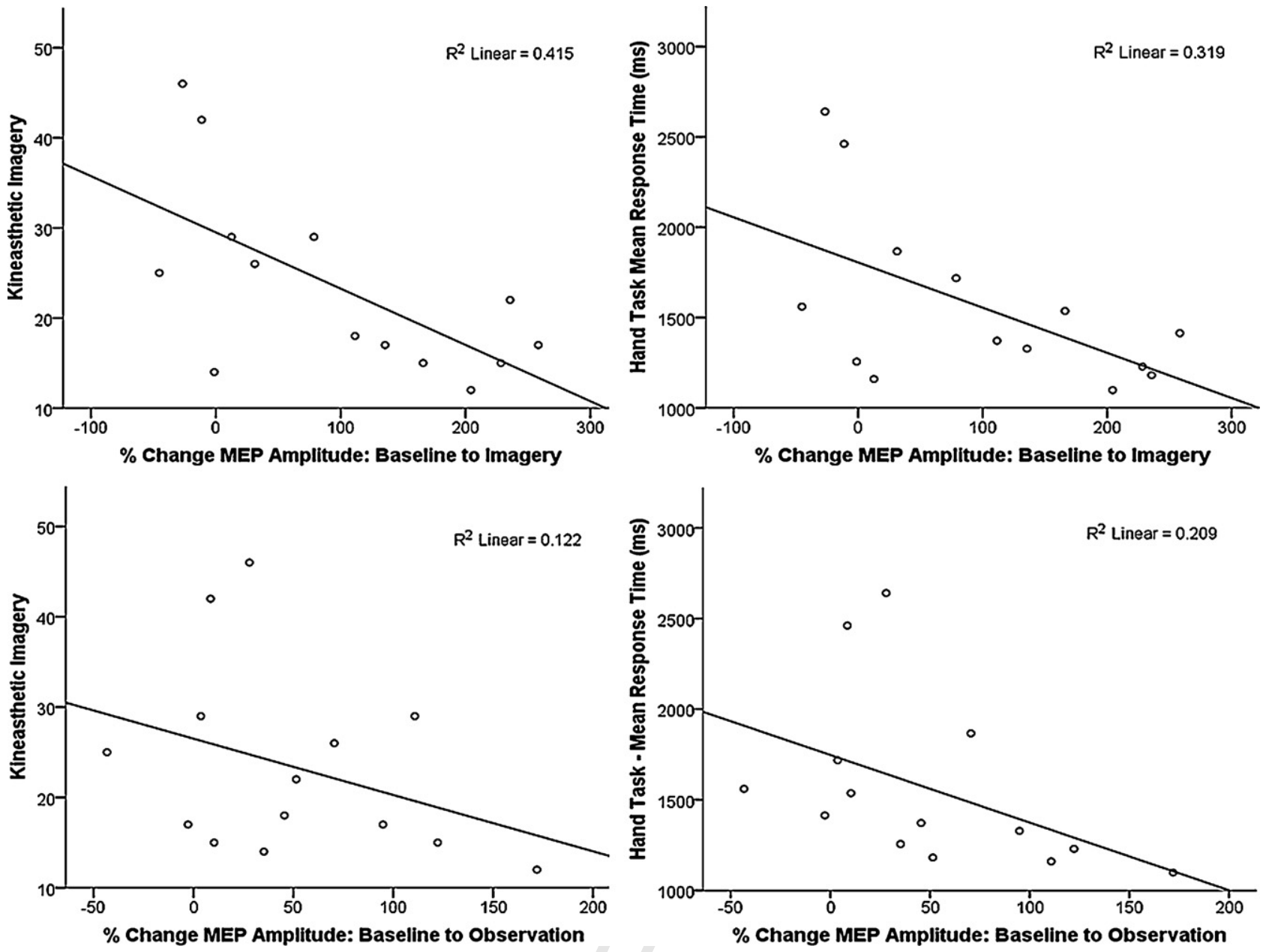

Fig. 4. Scatterplots demonstrating correlations between imagery ability measures and MEP change during imagery and observation conditions.

motor imagery and movement observation [e.g. 18-21], supporting the theoretical potential for imagery and observation intervention programs to facilitate recovery from conditions such as stroke and traumatic brain injury. As noted in the introduction, the conditions under which patients may benefit from imagery or observational training programs is not yet clearly understood.

\subsection{Correlations between imagery ability and MEP amplitude}

Change in MEP amplitude from baseline to imagery showed a strong, inverse correlation with the scores on the kinaesthetic component of the VMIQ-2 and hand task RT. This indicates that greater MEP amplitude was associated with more vivid kinaesthetic images and faster responses to hand stimuli and supports our hypothesis that activation of the motor system during motor imagery is likely to be influenced by motor imagery ability. This provides further evidence of disparity in neural activation between good and poor imagers, as shown during fMRI by Guillot et al. [15] and suggests that motor imagery ability may be a potential factor influencing the success of motor imagery training programs. This has implications for the use of imagery interventions in stroke patients, and may explain why the outcomes of such interventions are somewhat mixed [see 1 for a review].

The correlations between the change in MEP amplitude during imagery and the remaining imagery variables were less strong. There was a moderate correlation with internal visual imagery and no correlation with external visual imagery. This likely reflects the imagery mode and perspective utilised by participants in the study. When the TMS pulses were delivered, participants were seated with their hand on the table in front of them or in their lap, and were able to look at their hand during the task. As such, the utilisation of an external perspective would have been unlikely. This explains the poor correlation between MEP amplitude and external visual imagery scores. The correlation between visual internal imagery and MEP amplitude suggests that participants perhaps utilised a combination of internal visual and kinaesthetic imagery when imagining the finger-tapping movements. Hand task accuracy showed only a low-moderate correlation with MEP amplitude 
during imagery. This may be due to the ceiling effect on accuracy in this task (average accuracy was 90.5\%), which reduces the amount of variability observed.

Correlations between the motor imagery variables and change in MEP amplitude from baseline to the movement observation condition were not as strong as those using the imagery condition. There were only moderate, non-significant correlations between MEP amplitude change and kinaesthetic imagery, hand task RT and accuracy. This suggests that although both motor imagery and movement observation appear to facilitate activation of the corticospinal pathways to a similar extent, the activation during movement observation is less reliant on motor imagery ability. If future research demonstrates that the effectiveness of motor imagery training is mediated by initial motor imagery ability, movement observation training programs may provide a viable alternative that is less dependent on imagery ability. The benefits of movement observation training (where participants watch footage of motor tasks being performed) over motor imagery were recently reviewed by Holmes and Calmels [33]. These include better control of the image and the mode, perspective and agency employed. Observation also removes the need for complex imagery scripts and the possibility that the script is not completely followed.

While there has been speculation that motor imagery ability would mediate the effectiveness of motor imagery interventions, this study is only the second to show that neural activation during motor imagery is indeed influenced by motor imagery ability. This finding may be one of the reasons that reviews of the effectiveness of motor imagery training find mixed results $[1,34]$ and indicates that all clinical trials including a motor imagery intervention should include an evaluation of each participant's ability to form accurate and vivid movement images. The next step is to determine whether these findings can be replicated in a sample of older adults, more likely to suffer from stroke, and to determine the impact of these findings on motor imagery intervention outcomes. That is, does the reduced level of facilitation in the corticospinal pathway of people with lower imagery ability represent less effective motor imagery interventions? Along with this, it is crucial to determine if motor imagery interventions that improve imagery ability lead to increases in corticospinal facilitation, and whether patients whose imagery skills are impaired as a result of brain injury [e.g. those with parietal lesions; see, for example, 17] can regain those skills through imagery training. Furthermore, including a movement observation training program in studies aimed at answering these questions would provide significant advances to our current knowledge.

Although our findings regarding the relationship between MEP amplitude change from baseline to imagery and kinaesthetic imagery were quite strong, they were perhaps limited by our choice of measure and our lack of a clear imagery script for participants to follow during the finger tapping task. For example, had we asked participants to rate the vividness of their imagery during the tapping task, the correlation with MEP facilitation change may have been stronger - instead, we are making the assumption that their ability to produce vivid images as described by the VMIQ-2 reflected their ability to produce a vivid reproduction of the finger tapping task. Recent research has suggested that poor imagers may use different techniques to solve a visual, non-biological, imagery task to that used by good imagers [35] and as such, it would be recommended that imagery content be carefully monitored in future studies. Also, we could have provided clearer imagery scripts that asked participants to imagine the finger tapping task using both visual (internal and external) and kinaesthetic separately, and then examined the relationship between MEP amplitude during each reproduction with the individual components of the VMIQ-2.

\section{Conclusions}

Our findings lend support to those of Guillot et al. [15], suggesting that patterns of neural activation during motor imagery are related to the ability of the individual to produce vivid motor images. These are the first studies to identify these trends in average populations, with previous work focused on athletes imagining sport-specific skills. The results have important clinical applications given the increasing interest in the use of motor imagery as a tool for motor rehabilitation and raise a number of questions regarding the efficacy of these interventions for patients with poor imagery ability. Importantly, our data suggests that neural activation during movement observation is less related to imagery ability. Although this needs further exploration, it indicates that movement observation training programs may be a better alternative to imagery programs, particularly for those with a reduced ability to form vivid images. Finally, as a result of this research, we recommend that all studies examining the efficacy of motor imagery interventions include a measure of motor imagery ability in their test battery so that intervention results may be better understood.

\section{References}

[1] Sharma N, Pomeroy VM, Baron JC. Motor imagery: a backdoor to the motor system after stroke? Stroke 2006;37:1941-52.

[2] Simmons L, Sharma N, Baron JC, Pomeroy VM. Motor imagery to enhance recovery after subcortical stroke: who might benefit, daily dose, and potential effects. Neurorehabil Neural Repair 2008;22:458-67.

[3] Decety J, Grézes J. Neural mechanisms subserving the perception of human actions. Trends Cogn Sci 1999;3:172-8.

[4] Guillot A, Collet C, Nguyen VA, Malouin F, Richards C, Doyon J. Brain activity during visual versus kinesthetic imagery: an fMRI study. Hum Brain Mapp 2009;30:2157-72.

[5] Munzert J, Lorey B, Zentgraf K. Cognitive motor processes: the role of motor imagery in the study of motor representations. Brain Res Rev 2009;60:306-26.

[6] Clark S, Tremblay F, Ste-Marie D. Differential modulation of corticospinal excitability during observation, mental imagery and imitation of hand actions. Neuropsychologia 2003;42:105-12.

[7] Stinear CM, Byblow WD, Steyvers M, Levin O, Swinnen S. Kinesthetic, but not visual, motor imagery modulates corticomotor excitability. Exp Brain Res 2006;168:157-64.

[8] Ietswaart M, Johnston M, Dijkerman HC, Joice S, Scott CL, MacWalter RS, et al Mental practice with motor imagery in stroke recovery: randomized controlled trial of efficacy. Brain 2011;134:1373-86.

[9] Isaac AR, Marks DF. Individual differences in mental imagery experience: developmental changes and specialization. Br J Psychol 1994;85:479-500.

[10] Wei G, Luo J. Sport expert's motor imagery: functional imaging of professiona motor skills and simple motor skills. Brain Res 2010;1341:52-62.

[11] Chang Y, Lee J-J, Seo J-H, Song H-J, Kim Y-T, Lee HJ, et al. Neural correlates of motor imagery for elite archers. NMR Biomed 2011;24:366-72.

[12] Fourkas AD, Bonavolonta V, Avenanti A, Aglioti SM. Kinesthetic imagery and tool-specific modulation of corticospinal representations in expert tennis players. Cereb Cortex 2008;18:2382-90.

[13] Lacourse MG, Orr ELR, Cramer SC, Cohen MJ. Brain activation during execution and motor imagery of novel and skilled sequential hand movements. Neuroimage 2005;27:505-19.

[14] Hall CR, Martin KA. Measuring movement imagery abilities: a revision for the movement imagery questionnaire. J Ment Imagery 1997;21:143-54.

[15] Guillot A, Collet C, Nguyen VA, Malouin F, Richards C, Doyon J. Functional neuroanatomical networks associated with expertise in motor imagery. Neuroimage 2008;41:1471-83.

[16] Lotze M, Halsband U. Motor imagery. J Physiol 2006;99:363-95.

[17] Sirigu A, Duhamel JR, Cohen L, Pillon B, Dubois B, Agid Y. The mental representation of hand movements after parietal cortex damage. Science 1996;273:1564-8

[18] Fadiga L, Buccino G, Craighero L, Fogassi L, Gallese V, Pavesi G. Corticospinal excitability is specifically modulated by motor imagery: a magnetic stimulation study. Neuropsychologia 1999;37:147-58.

[19] Fourkas AD, Avenanti A, Urgesi C, Aglioti SM. Corticospinal facilitation during first and third person imagery. Exp Brain Res 2006;168:143-51.

[20] Voisin JIA, Mercier C, Jackson PL, Richards C, Malouin F. Is somatosensory excitability more affected by the perspective or modality content of motor imagery? Neurosci Lett 2011;493:33-7.

[21] Roosink M, Zijdewind I. Corticospinal excitability during observation and imagery of simple and complex hand tasks: implications for motor rehabilitation. Behav Brain Res 2010;213:35-41.

[22] Roberts R, Callow N, Hardy L, Markland D, Bringer J. Movement imagery ability: development and assessment of a revised version of the Vividness and Movement Imagery Questionnaire. J Sport Exerc Psychol 2008;30:200-21. 
[23] Williams J, Anderson V, Reddihough DS, Reid SM, Vijayakumar N, Wilson PH. A comparison of motor imagery performance in children with spastic hemiplegia and developmental coordination disorder. J Clin Exp Neuropsychol 2010, doi:10.1080/13803395.2010.509714.

[24] Oldfield RC. The assessment and analysis of handedness: the Edinburgh Inventory. Neuropsychologia 1971;9:97-113.

[25] McCarron LT. McCarron assessment of neuromuscular development: fine and gross motor abilities. revised ed. Dallas, TX: Common Market Press; 1997.

[26] Mercier C, Aballea A, Vargas CD, Paillard J, Sirigu A. Vision without proprioception modulates cortico-spinal excitability during hand motor imagery. Cereb Cortex 2008;18:272-7.

[27] Cohen J. Statistical power analysis for the behavioral sciences. 2nd ed. New Jersey: Lawrence Erlbaum; 1988.

[28] Parsons LM. Imagined spatial transformations of one's hands and feet. Cognit Psychol 1987;19:178-241.
[29] Kosslyn SM, Digirolamo GJ, Thompson WL, Alpert NM. Mental rotation of objects versus hands: neural mechanisms revealed by positron emission tomography. Psychophysiology 1998;35:151-61.

[30] Parsons LM, Fox PT. The neural basis of implicit movements used in recognising hand shape. Cogn Neuropsychol 1998;15:583-615.

[31] Deconinck FJA, Spitaels L, Fias W, Lenoir M. Is developmental coordination disorder a motor imagery deficit? J Clin Exp Neuropsychol 2009;31:720-30.

[32] Williams J, Reid SM, Reddihough DS, Anderson V. Motor imagery ability of children with congenital hemiplegia: effect of lesion side and functional level. Res Dev Disabil 2011;32:740-8.

[33] Holmes PS, Calmels C. A neuroscientific review of imagery and observation use in sport. J Motor Behav 2008;40:433-45.

[34] Mulder T. Motor imagery and action observation: cognitive tools for rehabilitation. J Neural Transm 2007;114:1265-78.

[35] Logie RH, Pernet CR, Buonocore A, Della Sala S. Low and high imagers activate networks differentially in mental rotation. Neuropsychologia 2011;49:3071-7. 